\title{
Leistungs- und Abrechnungsdatum in GKV-Routinedaten: Umgang mit zeitlichen Abweichungen
}

\section{Date of Service Provision and Date of Payment in Claims Data: Dealing with Time Differences}

\section{(우(1) $\odot \odot$}

\author{
Autoren \\ Bianka Ditscheid $^{1 *}$, Josephine Storch ${ }^{1}{ }^{*}$, Markus Krause ${ }^{1}$, Ingo Meyer $^{2}$, Antje Freytag ${ }^{1}$
}

Institute

1 Institut für Allgemeinmedizin, Universitätsklinikum Jena, Jena

2 PMV forschungsgruppe, Uniklinik Köln, Köln

Schlüsselwörter

GKV-Routinedaten, medizinische Inanspruchnahme, Beobachtungszeitraum, Leistungs-/Abrechnungsdatum, Tod

Key words

Claims data, health services utilization, observation period, date of service provision/payment, death, administrative claims

\section{Bibliografie}

DOI https://doi.org/10.1055/a-1030-4223

Online-Publikation: 10.12.2019

Gesundheitswesen 2020; 82 (Suppl. 1): S20-S28

(c) Georg Thieme Verlag KG Stuttgart · New York

ISSN 0949-7013

Korrespondenzadresse

Dr. Antje Freytag

Institut für Allgemeinmedizin

Universitätsklinikum Jena

Bachstraße 18

07743 Jena

Antje.Freytag@med.uni-jena.de

\section{ZUSAMMENFASSUNC}

Ziel Ziel dieser Untersuchung ist es, das aus dem Auseinanderfallen von Leistungs- und Abrechnungsdatum in Krankenkassenroutinedaten resultierende Risiko von Fehleinschätzungen der Inanspruchnahme in verschiedenen Leistungsbereichen (ambulante ärztliche Behandlung, Arzneimittel, Heil- und Hilfsmittel, Krankenhaus) in Bezug zum Tod als Indexereignis sowie in Bezug zum Jahreswechsel zu quantifizieren, Erklärungsansätze darzulegen und mögliche Lösungsstrategien aufzuzeigen.

Die erst- und zweitgenannte Autorin trugen zu gleichen Teilen bei.
Methodik In einem Wissenschaftlichen Data Warehouse einer großen deutschen Krankenkasse standen vorstrukturierte Daten von in 2016 verstorbenen Versicherten zur Verfügung. Die Darstellung zeitlicher Abweichungen von Leistungs- und Abrechnungsdaten erfolgte für verschiedene ambulante und stationäre Versorgungsbereiche unter Heranziehung von Daten der Jahre 2015 bis 2017. Ein exaktes Abrechnungsdatum (TT/MM/JJJ) war lediglich bei Heil- und Hilfsmittelverordnungen verfügbar. Bei Arzneimittel-Verordnungen war das Abrechnungsdatum lediglich monatsgenau (MM/JJJ). Bei ambulanten ärztlichen Leistungen lag nur das Abrechnungsquartal vor (Q/JJJJ).

Ergebnisse Die Analysen zeigen, dass im Leistungsbereich der ambulanten ärztlichen Behandlung und der Krankenhausbehandlung < 1 \% der Fälle/Gebührenordnungspositionen (GOP)/OPS nach dem Tod abgerechnet wurden, im Bereich der Heil- und Hilfsmittel jedoch 28-31\% der Verordnungen (VO). Ein Großteil der Leistungsabrechnungen nach dem Tod erfolgte innerhalb von 3 Monaten (93\% der Heil- und Hilfsmittel, 67\% der ambulanten ärztlichen Behandlungsfälle). Über den Jahreswechsel 2015/2016 zeigte sich eine zeitliche Differenz bei < $1 \%$ der ambulanten GOP bis hin zu $18 \%$ bei den Heilmittel-VO. Auch hier wurde die Mehrzahl der Leistungen innerhalb der ersten 3 Monate nach dem Jahreswechsel abgerechnet (100\% der Heil- und Hilfsmittel, $65 \%$ der ambulanten GOP).

Schlussfolgerung Der zeitliche Verzug zwischen Leistungsund Abrechnungsdaten stellt bei Analysen der GKV-Leistungsinanspruchnahme bis zum Tod eines Versicherten sowie beim Jahreswechsel eine Herausforderung dar und ist eine Quelle für Unter-/Überschätzungsfehler. So ist bei Datenanforderung, -aufbereitung und -auswertung explizit darauf zu achten, dass auch noch nach dem zu betrachtenden Zeitraum abgerechnete Leistungen berücksichtigt werden.

\section{ABSTRACT}

Aim The paper quantifies discrepancies between date of payment and date of service provision when doing analyses in relation to date of death and also in relation to the end of a calendar year. In analyses of this type, time differences between service provision and payment can lead to both under- and overestimation of service use. We aim to capture these pheno- 
mena in claims data from different sectors (primary care, medication prescription, prescription of remedies and medical aids, hospital care).

Method We have used pre-structured claims data from a scientific data warehouse of a large German statutory health insurance covering people that died in 2016 . We investigated the discrepancies in time between date of service provision and date of payment for different outpatient and inpatient services based on data from 2015 to 2017. An exact date (dd/mm/yyyy) was only available for data covering prescriptions of remedies and medical aids. Data covering medication prescriptions were only exact to the month of payment (mm/yyyy), whereas data covering outpatient physician care were only exact to the quarter of payment (q/yyyy).

Results For both outpatient physician care and hospital care, less than $1 \%$ had a payment date after the date of death. The share is considerably higher (28-31\%) for prescriptions of remedies and medical aids. The majority of payments occurred within 3 months after death ( $93 \%$ for prescriptions of remedies and medical aids, $67 \%$ for primary care services). Less than $1 \%$ of outpatient physician care and about $18 \%$ of remedies had been paid after the end of the calender year 2015 . Here too, the majority of payments were made within the first 3 months of 2016 ( $100 \%$ of prescriptions of remedies and medical aids, $65 \%$ of primary care services).

Conclusions Discrepancies in time between date of service provision and date of payment pose a challenge and are a potential source of under-/overestimation of health service utilization when doing analyses in relation to date of death or the end of a calendar year. This needs to be taken into account when requesting the data, but also in preparing and analysing them. The primary recommendation is to ensure that services with a payment date after death are included explicitly.

\section{Einleitung}

Die Ermittlung der Leistungsinanspruchnahme von Versicherten der gesetzlichen Krankenkassen (GKV) in einem definierten Beobachtungszeitraum ist keine triviale Aufgabe. Die zeitraumbezogene Zuordnung von Leistungen kann sich je nach verwendeter Datenquelle und betreffendem Versorgungsbereich unterscheiden. Während die Daten in einem Wissenschaftlichen Data Warehouse (WDWH) bereits vorstrukturiert sind, können sie im Rahmen einer projektbezogenen Datenanforderung (minimal data set) der Fragestellung einer Studie entsprechend definiert werden.

In der Regel gilt, dass die zur Verfügung gestellten Daten ausschließlich bereits abgerechnete Leistungen enthalten. Der zeitliche Verzug bei der Abrechnung von Leistungen bestimmt somit wesentlich die Verfügbarkeit dieser Leistungen in den Abrechnungsdaten der GKV. Zusätzlich ist zu beachten, dass die Daten auch bei der jeweiligen Krankenkasse mit einer zeitlichen Verzögerung vorliegen. Diese kann bis zu acht Monate nach Quartalsende (z. B. bei vertragsärztlichen Leistungen) betragen [1].

In den bereitgestellten Daten können, abhängig von den verwendeten Datenstrukturen der jeweiligen Krankenkasse, nur das Abrechnungsdatum, nur das Leistungsdatum oder beide Angaben enthalten sein. Für Analysen der Inanspruchnahme ist das Leistungsdatum bzw. der Leistungszeitraum die geeignetere Kennzahl. Der Abrechnungszeitpunkt liegt natürlicherweise hinter dem Leistungszeitpunkt bzw. dem Ende eines Leistungszeitraums. Diese zeitliche Differenz zwischen Leistungs- und Abrechnungsdatum birgt sowohl bei Analysen bis zu einem Indexereignis, z. B. dem Tod eines Versicherten (VS), als auch bei jahresbezogenen Beobachtungszeiträumen die Gefahr der Fehlschätzung der Inanspruchnahme ( $\mathbf{A b b} \mathbf{1}$ ).

Unseres Wissens liegen bisher keine Ergebnisse zur exemplarischen Quantifizierung der Abweichungen von Leistungs- und Abrechnungsdatum für Deutschland vor. Insbesondere in Bezug zum Tod, zu (anderen) versichertenindividuellen Zeitpunkten oder dem Jahreswechsel als Ende eines Beobachtungszeitraums ist es notwendig, sich dies bewusst zu machen und eine Strategie zum Umgang in den eigenen Analysen zu entwickeln.
Bisher finden sich in der Literatur lediglich Hinweise darauf, wie es zu zeitlichen Differenzen zwischen Abrechnungs- und Leistungsdatum bzw. Leistungszeitraum kommen kann. So weisen Horenkamp-Sonntag et al. im Kapitel „Prüfung, Datenqualität und Validität von GKV-Routinedaten “ darauf hin, dass es aus unterschiedlichen Gründen zu zeitlichen Implausibilitäten kommen kann und diese gemäß der Guten Praxis Sekundärdatenanalyse [2] geprüft und je nach Fragestellung berücksichtigt werden sollten [3].

Ziel dieser Untersuchung ist es daher, das aus dem Auseinanderfallen von Leistungs- und Abrechnungsdatum in Krankenkassenroutinedaten resultierende Risiko von Fehleinschätzungen der Inanspruchnahme in verschiedenen Leistungsbereichen in Bezug zum Tod als Indexereignis sowie in Bezug zum Jahreswechsel zu quantifizieren, Erklärungsansätze für nach dem Tod abgerechnete Leistungen darzulegen und mögliche Lösungsstrategien aufzuzeigen.

\section{Methodik}

Wir führten eine Analyse zeitlicher Differenzen zwischen Leistungsinanspruchnahme und -abrechnung im Hinblick auf 2 Zeitpunkte durch: das Todesdatum sowie den Jahreswechsel 2015/2016.

Die Analysen mit Bezug zum Todesdatum wurden differenziert nach (a) Abrechnungen nach dem Tod, (b) Leistungen nach dem Tod sowie (c) Leistungen in den letzten 6 Lebensmonaten mit Abrechnung nach dem Tod. Die analysierten Leistungsbereiche umfassten dabei ambulante Behandlungsfälle und die entsprechenden Gebührenordnungspositionen (GOP), ambulante Verordnungen (VO) von Arzneimitteln (AM), Heil- und Hilfsmitteln sowie Krankenhausfälle und stationär durchgeführte Operationen/Prozeduren (OPS).

Da das Todesdatum in den uns zur Verfügung stehenden Abrechnungsdaten nicht als vordefiniertes Datenfeld existierte, wurde es aus dem Datum des Versicherungsendes mit dem Tod als Austrittsgrund aus der Krankenkasse abgeleitet. Die den Analysen zugrunde liegende Population ist Teil einer Studienpopulation, die für ein laufendes Forschungsprojekt selektiert wurde: Für dieses 


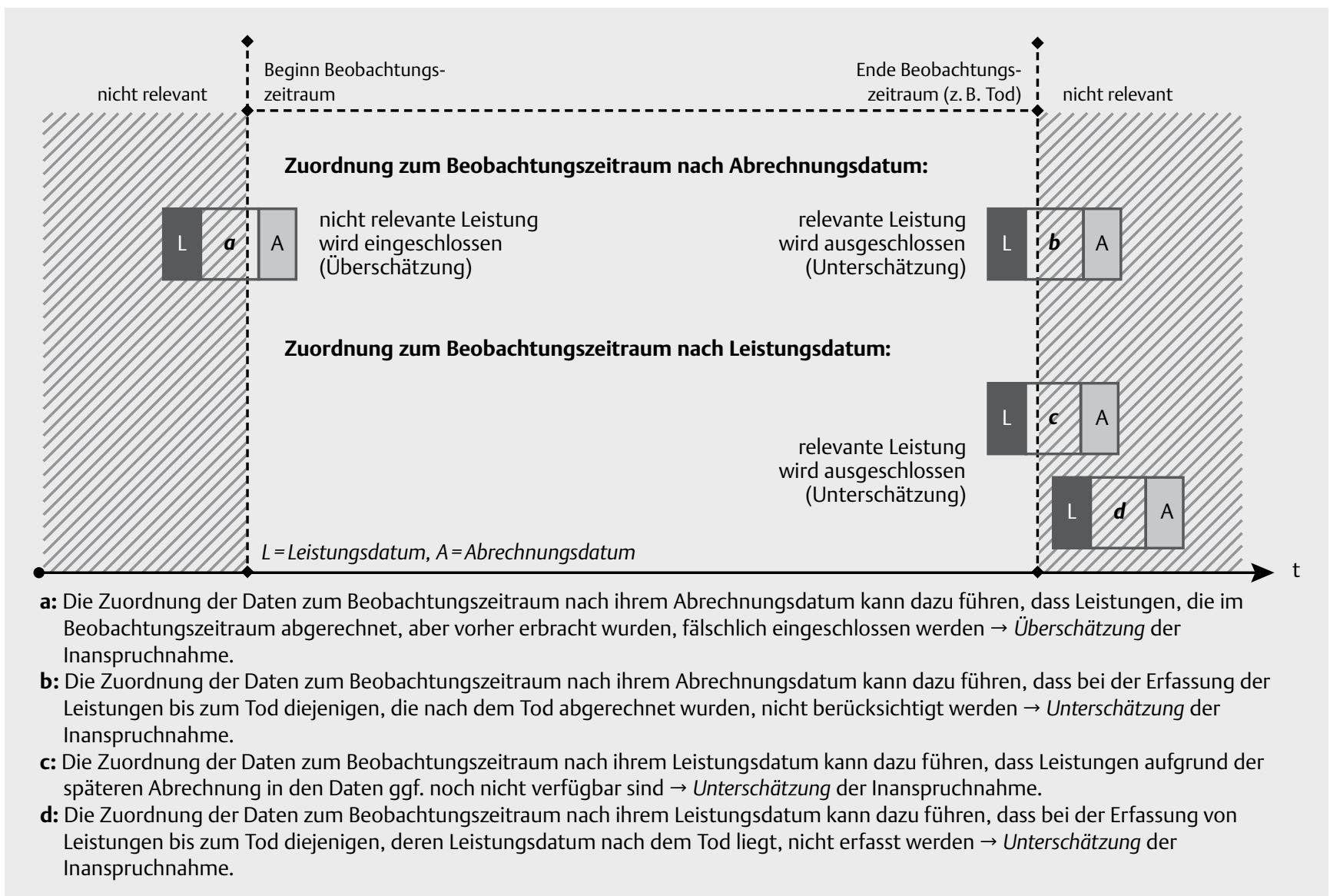

Abb. 1 Zuordnung von Leistungen zum Beobachtungszeitraum und resultierende Fehlschätzung.

wurden Versicherte herangezogen, die ein Todesdatum im Jahr 2016 hatten, mindestens 2 Jahre vor ihrem Tod durchgängig bei der datenliefernden Krankenkasse versichert und zum Zeitpunkt des Todes mindestens 19 Jahre alt waren. Für unsere Analysen wurden Versicherte eingeschlossen, die im Zeitraum Juli bis Dezember 2016 verstorben waren $(N=47610)$. Ausgewertet wurden die jeweiligen Abrechnungsdatensätze der Jahre 2015, 2016 und 2017.

Auf Grundlage der in den jeweiligen Leistungsbereichen unterschiedlich ausgeprägten Leistungs- und Abrechnungszeiträume haben wir folgende Definitionen für die Leistungsinanspruchnahme und Abrechnung formuliert: Als Zeitpunkt der Abrechnung wurde das Abrechnungsquartal für (1) ambulante GOP und (2) ambulante Behandlungsfälle, (3) der Abrechnungsmonat für ambulante Arzneimittelverordnungen (Arzneimittel-VO), (4) das Abrechnungsdatum bei Heil- und Hilfsmittelverordnungen (Heilmittel-VO, Hilfsmittel-VO) und das Entlassungsdatum des stationären Behandlungsfalls bei (5) Krankenhausfällen und (6) stationären OPS festgelegt ( $\triangleright$ Tab. 1). Als eine Abrechnung nach dem Tod wurde entsprechend die Abrechnung eines ambulanten Falls ab dem Quartal nach dem Todesquartal für (1) ambulante GOP und (2) ambulante Fälle definiert. Für (3) ambulante Arzneimittel-VO wurde die Abrechnung ab dem Monat nach dem Todesmonat und für (4) ambulante Heil- und Hilfsmittel-VO das Abrechnungsdatum nach dem Todesdatum herangezogen. Für (5) Krankenhausfälle und (6) stationäre OPS wurde - weil das Abrechnungsdatum des Falls in den
Daten nicht verfügbar war - ein Entlassungsdatum nach dem Todesdatum als Abrechnung nach dem Tod definiert ( $\triangleright$ Tab. 1).

Als Zeitpunkt der Leistungsinanspruchnahme wurde (1) das Leistungsdatum für ambulante GOP, (2) das Ende der Behandlung für ambulante vertragsärztliche Behandlungsfälle, (3) das Datum der Arzneimittelabgabe in der Apotheke für ambulante Arzneimittel-VO, (4) das Ende der Leistung für Heil- oder Hilfsmittel-VO, (5) das Entlassungsdatum als Behandlungsende eines Krankenhausfalls und (6) das Leistungsdatum einer stationären OPS definiert ( tung nach dem Tod wurde entsprechend definiert: (1) ein Leistungsdatum einer ambulanten GOP nach dem Todesdatum, (2) ein nach dem Todesdatum endender ambulanter Behandlungsfall, (3) eine Arzneimittelabgabe nach dem Todesdatum, (4) ein Leistungsendedatum für Heil- oder Hilfsmittel nach dem Todesdatum, (5) ein Entlassungsdatum nach dem Todesdatum für Krankenhausfälle und (6) für stationäre OPS ein Leistungsdatum nach dem Todesdatum ( $\triangleright$ Tab. 1).

Im Ergebnis wurden bei der Betrachtung mit Bezug zum Todesdatum jeweils Anteil und Anzahl der Leistungen, die nach dem Tod abgerechnet wurden, sowie Anteil und Anzahl der Versicherten mit entsprechenden Leistungen in unserer Studienpopulation ausgewiesen. Dabei beziehen sich die Angaben zum Anteil der Versicherten immer auf die Grundgesamtheit der Studienpopulation ( $N=47610)$. Die Angaben zum Anteil der Leistungsarten (GOP, ambulante Fälle, VO, Krankenhausfälle, OPS) beziehen sich immer auf die Summe der in den letzten 6 Lebensmonaten vor dem Todes- 
> Tab. 1 Definition der zeitlichen Bezüge für Leistungs- und Abrechnungszeitpunkte.

\begin{tabular}{|l|l|l|l|l|l|l|}
\hline & Ambulante GOP & Ambulante Fälle & Arzneimittel-VO & Heil- und Hilfsmittel- Vo & Krankenhaus-Fälle & Stationäre OPS \\
\hline $\begin{array}{l}\text { Abrechnungs- } \\
\text { zeitpunkt }\end{array}$ & \multicolumn{2}{|l|}{ Ende des Abrechnungsquartals } & $\begin{array}{l}\text { Ende des } \\
\text { Abrechnungs- } \\
\text { monats }\end{array}$ & Abrechnungsdatum & Entlassungsdatum des stationären Falls \\
\hline $\begin{array}{l}\text { Abrechnung } \\
\text { nach Tod }\end{array}$ & $\begin{array}{l}\text { Abrechnung im Folgequartal nach } \\
\text { Sterbequartal }\end{array}$ & $\begin{array}{l}\text { Abrechnung im } \\
\text { Folgemonat nach } \\
\text { Todesmonat }\end{array}$ & $\begin{array}{l}\text { Abrechnungsdatum nach } \\
\text { Todesdatum }\end{array}$ & Entlassungsdatum nach dem Todesdatum \\
\hline $\begin{array}{l}\text { Leistungs- } \\
\text { zeitpunkt }\end{array}$ & $\begin{array}{l}\text { Leistungsdatum für } \\
\text { ambulante GOP }\end{array}$ & $\begin{array}{l}\text { Ende des } \\
\text { Behandlungsfalls }\end{array}$ & $\begin{array}{l}\text { Datum der } \\
\text { Arzneimittel- } \\
\text { abgabe }\end{array}$ & Ende der Leistung & Entlassungsdatum & Leistungsdatum \\
\hline $\begin{array}{l}\text { Leistung nach } \\
\text { Tod }\end{array}$ & $\begin{array}{l}\text { Leistungsdatum } \\
\text { einer ambulanten } \\
\text { GOP nach dem } \\
\text { Todesdatum }\end{array}$ & $\begin{array}{l}\text { Ende des } \\
\text { Behandlungsfalls } \\
\text { nach dem } \\
\text { Todesdatum }\end{array}$ & $\begin{array}{l}\text { Arzneimittelabga- } \\
\text { be nach dem } \\
\text { Todesdatum }\end{array}$ & $\begin{array}{l}\text { Ende der Leistung nach } \\
\text { dem Tod }\end{array}$ & $\begin{array}{l}\text { Entlassungsdatum } \\
\text { nach dem } \\
\text { Todesdatum }\end{array}$ & $\begin{array}{l}\text { Leistungsdatum } \\
\text { nach dem } \\
\text { Todesdatum }\end{array}$ \\
\hline
\end{tabular}

datum (für Heil- und Hilfsmittel, Krankenhausfälle, stationäre OPS) bzw. vor dem Todesmonat (für AM-VO) bzw. vor dem Todesquartal (für ambulante ärztliche Leistungen) erbrachten bzw. abgerechneten Leistungen.

Bei der Betrachtung mit Bezug zum Jahreswechsel 2015/2016 basieren die Angaben zum Anteil der Versicherten auf der Anzahl derjenigen Versicherten, die tatsächlich Leistungen aus dem jeweiligen Leistungsbereich in 2015 in Anspruch genommen haben. Die Angaben zum Anteil der Leistungsarten (GOP, ambulante Fälle, VO, OPS), die erst nach dem Jahreswechsel 2015/2016 abgerechnet wurden, beziehen sich auf die Summe der in 2015 bis zum Jahresende erbrachten Leistungen. Für Krankenhausfälle sind diese Berechnungen auf unserer Datenbasis nicht möglich, da Leistungsund Abrechnungsdatum identisch sind.

\section{Ergebnisse}

Die Ergebnisse sind für alle Leistungsbereiche und die beiden Zeitpunkte Todesdatum und Jahreswechsel 2015/2016 in > Tab. 2 dar gestellt.

\section{Abrechnungen nach dem Tod}

Im Leistungsbereich der ambulanten vertragsärztlichen Versorgung wurden - bezogen auf die in den letzten 6 Lebensmonaten abgerechneten Leistungen - 0,4\% der GOP und 0,5\% der Behandlungsfälle nach dem Tod abgerechnet. Am höchsten war der Anteil der nach dem Tod abgerechneten Leistungen bei Heilmitteln (31,1\%), gefolgt von Hilfsmitteln (28,2\%). Arzneimittel-VO wurden zu 1,1\% und Krankenhausfälle sowie stationäre OPS zu jeweils $0,2 \%$ nach dem Tod abgerechnet. Bezogen auf unsere Studienpopulation betraf dies zwischen 0,3\% (Krankenhausfälle, stationäre OPS) und 57,6\% (Hilfsmittel) der Versicherten ( $>$ Tab. 2, Zeile 1.1). Von diesen nach dem Tod abgerechneten Leistungen wurde der überwiegende Teil in den 3 Monaten bzw. im Folgequartal nach dem Todesdatum abgerechnet ( $\triangleright$ Tab. 2, Zeile 1.2).

\section{Leistungen nach dem Tod}

Für die Inanspruchnahme von Leistungen nach dem Tod, für die ein konkretes Datum herangezogen werden konnte, ergibt sich - bezogen auf die in den letzten 6 Lebensmonaten in Anspruch genommenen Leistungen - ein etwas anderes Bild: anteilig war die Leistungs- inanspruchnahme bei Hilfsmitteln mit 9,8\% am höchsten, gefolgt von ambulanten ärztlichen Behandlungsfällen mit 1,6\%, Arzneimittel-VO mit 0,8\% und ambulanten GOP mit 0,3\% der Leistungen. Für die Inanspruchnahme nach dem Tod wurden in den Leistungsbereichen Krankenhausfälle ( $0,2 \%)$, Heilmittel $(0,1 \%)$ und stationäre OPS $(0,02 \%)$ die geringsten Anteile ermittelt. Bezogen auf unsere Studienpopulation betraf dies zwischen 0,1 \% (stationäre OPS) und 39, 1 \% (Hilfsmittel) der Versicherten ( $>$ Tab. 2, Zeile 2.1). Der überwiegende Teil der nach dem Tod erbrachten Leistungen fällt in den Zeitraum von 3 Monaten nach dem Tod ( $\triangleright$ Tab. 2, Zeile 2.2).

Es wirkt zunächst inkonsistent, dass unsere Berechnungen für ambulante Fälle mehr Leistungen ( $\triangleright$ Tab. 2, Zeile 2.1) als Abrechnungen nach dem Tod ( $\triangleright$ Tab. 2, Zeile 1.1) ergaben. Für die Ermittlung der Leistungen nach dem Tod konnte ein konkretes Datum (Behandlungsende des ambulanten Falls; > Tab. 1) herangezogen werden, während für Abrechnungen nach dem Tod mit Quartalsdifferenzen in Bezug zum Tod gerechnet wurde, da hier lediglich das Abrechnungsquartal verfügbar war. Dies führt zu einer Unterschätzung der Abrechnungen nach dem Tod in diesem Leistungsbereich.

\section{Leistungen in den letzten 6 Lebensmonaten mit Abrechnung nach dem Tod}

Von den Leistungen mit Leistungsdatum innerhalb von 6 Monaten vor dem Tod wurden 0,2\% der stationären OPS, 0,3\% der ambulanten Fälle, 0,4\% der ambulanten GOP und 1,0\% der Arzneimittel-VO erst nach dem Tod abgerechnet. Bei den Heil- und Hilfsmitteln waren die Anteile mit 35,8 und 25,1\% deutlich höher. Bezogen auf unsere Studienpopulation betraf dies zwischen 0,3\% (stationäre OPS) und 48,3\% der Versicherten (Hilfsmittel; > Tab. 2, Zeile 4.1). Der überwiegende Teil der Leistungen wurde in den folgenden 3 Monaten bzw. im Folgequartal nach dem Versterben abgerechnet ( $\triangleright$ Tab. 2, Zeile 4.2). Lediglich jeweils 0,1\% der ambulanten GOP, der ambulanten Fälle sowie der Arzneimittel-VO wurden erst später als 3 Monate (bzw. nach dem ersten Quartal) nach dem Tod abgerechnet. Im Gegensatz dazu wurden im Leistungsbereich der Heilund Hilfsmittel bis zu 2,3\% der VO in einem Zeitraum von 3 Monaten nach dem Tod noch nicht abgerechnet ( $\triangleright$ Tab. 2, Zeile 4.3).

\section{Jahreswechsel 2015/2016}

Das Auseinanderfallen von Leistungs- und Abrechnungsdatum ist auch über den Jahreswechsel 2015/2016 hinweg in allen Leistungs- 


\begin{tabular}{|c|c|c|c|c|c|c|c|c|c|c|}
\hline $\begin{array}{l}\tilde{a} \\
0 \\
\varrho\end{array}$ & $\begin{array}{l}\bar{\Xi} \\
\grave{o} \\
\check{s}\end{array}$ & 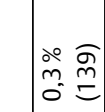 & $\begin{array}{l}\stackrel{\circ}{\circ} \\
\stackrel{\circ}{\circ} \\
\stackrel{\rho}{=}\end{array}$ & 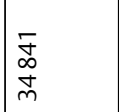 & 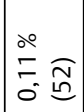 & $\begin{array}{l}\stackrel{\circ}{0} \\
\stackrel{0}{\circ} \\
0\end{array}$ & 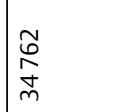 & 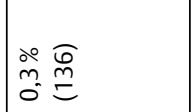 & $\begin{array}{l}\stackrel{\circ}{\circ} \\
\stackrel{0}{\circ} \\
\stackrel{0}{0}\end{array}$ & $\begin{array}{l}0 \\
\stackrel{0}{0} \\
o \\
0 \\
0\end{array}$ \\
\hline 吾 & $\begin{array}{l}\stackrel{0}{0} \\
\check{0} \\
\vdots\end{array}$ & 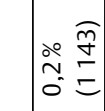 & 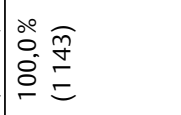 & 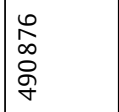 & $\begin{array}{l}\stackrel{0}{0} \\
\stackrel{0}{0} \\
0 \\
0\end{array}$ & $\begin{array}{l}\stackrel{\circ}{0} \\
\stackrel{0}{\circ} \overline{\bar{\infty}}\end{array}$ & 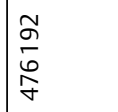 & 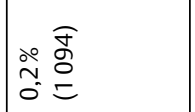 & $\begin{array}{l}\stackrel{0}{0} \\
\stackrel{\circ}{\circ} \\
\stackrel{\circ}{\circ}=\end{array}$ & $\stackrel{\circ}{\circ} 0$ \\
\hline 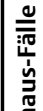 & $\stackrel{\circ}{\check{s} \Xi}$ & 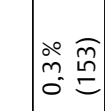 & 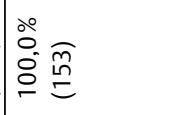 & \begin{tabular}{|l}
9 \\
$\mathscr{2}$ \\
0 \\
0 \\
0
\end{tabular} & 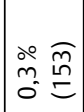 & 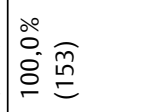 & $\begin{array}{l}2 \\
0 \\
0 \\
0 \\
m\end{array}$ & 京 & $\stackrel{\overrightarrow{\dot{C}}}{\mathrm{E}}$ & $\vec{i}$ \\
\hline 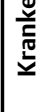 & 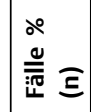 & 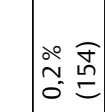 & 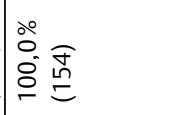 & \begin{tabular}{l}
\multirow{J}{N}{} \\
న్ \\
$\infty$
\end{tabular} & 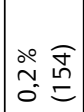 & 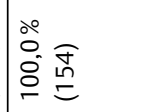 & $\begin{array}{l} \pm \\
\\
\infty \\
\infty\end{array}$ & 主 & $\stackrel{\vec{C}}{\check{c}}$ & $\vec{i}$ \\
\hline $\bar{\Xi}$ & $\begin{array}{l}\stackrel{\circ}{s} \\
\stackrel{s}{s} \Xi\end{array}$ & 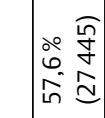 & $\begin{array}{ll} & 0 \\
\circ & 0 \\
N & 0 \\
& 0\end{array}$ & 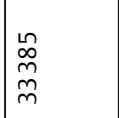 & 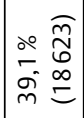 & $\begin{array}{l}\stackrel{\widehat{D}}{\circ} \\
\stackrel{\infty}{\infty} \\
\tilde{\sigma}^{\infty} \\
\sigma\end{array}$ & 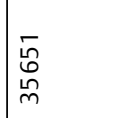 & 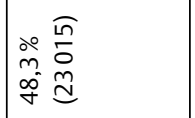 & 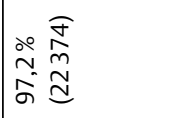 & $\begin{array}{l}\stackrel{\circ}{\circ} \\
\stackrel{0}{\circ}=\end{array}$ \\
\hline 訔 & $\stackrel{\circ}{\rho}$ ఏ & $\begin{array}{ll} & \widehat{\alpha} \\
\stackrel{0}{\sim} & 0 \\
\sim & 0 \\
\infty & 0 \\
\sim & 0\end{array}$ & 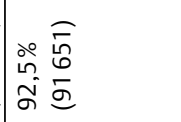 & 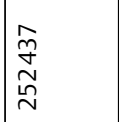 & $\begin{array}{ll} & \overline{\bar{\sigma}} \\
0 & \bar{\delta} \\
\infty & 0 \\
\sigma & \overline{\tilde{n}}\end{array}$ & 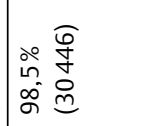 & $\begin{array}{l}\infty \\
\infty \\
\infty \\
\infty \\
\sim \\
\sim\end{array}$ & 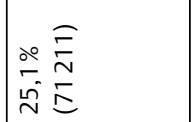 & 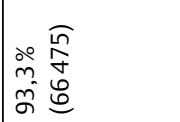 & 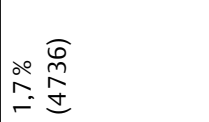 \\
\hline $\bar{\Phi}$ & $\begin{array}{l}\circ \\
\sim \\
\sim\end{array}$ & 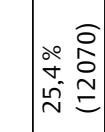 & 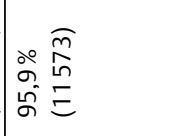 & $\begin{array}{l}\frac{6}{2} \\
\frac{1}{n}\end{array}$ & $\begin{array}{l}\hat{\overline{5}} \\
\stackrel{0}{\circ} \\
\overline{0}\end{array}$ & $\begin{array}{l}\stackrel{\circ}{\hat{n}} \widehat{\Omega} \\
\bar{\sigma}\end{array}$ & 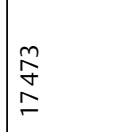 & 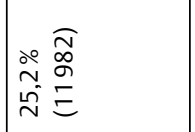 & 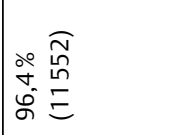 & 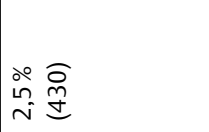 \\
\hline$\overline{\underline{T}}$ & $\stackrel{\circ}{\rho} \Xi$ & 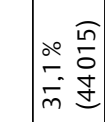 & 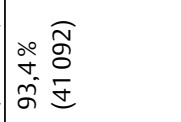 & 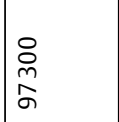 & 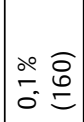 & $\begin{array}{l}\stackrel{\circ}{\circ} \\
\infty \\
\text { ñ }\end{array}$ & 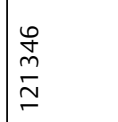 & 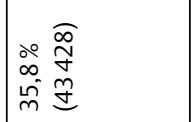 & 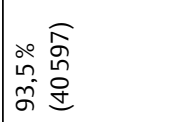 & 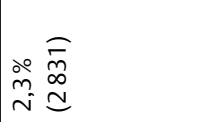 \\
\hline $\bar{g}$ & $\stackrel{\circ}{\check{s} \Xi}$ & 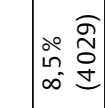 & 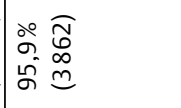 & 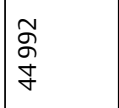 & 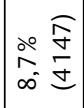 & 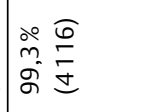 & 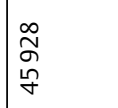 & 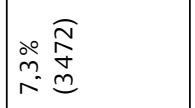 & 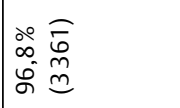 & $\mid \begin{array}{l}\text { ơ } \\
\text { Ñ. } \\
\text { o. }\end{array}$ \\
\hline$\frac{\mathscr{V}}{\frac{N}{4}}$ & $\stackrel{\circ}{\rho} \Xi$ & 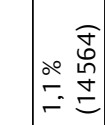 & 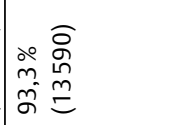 & $\begin{array}{l}0 \\
0 \\
0 \\
\infty \\
0 \\
m \\
-1\end{array}$ & 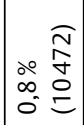 & 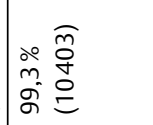 & 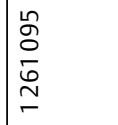 & 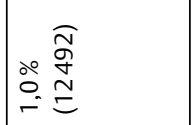 & 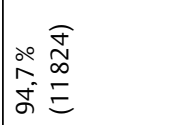 & $\begin{array}{l}\stackrel{0}{\widehat{o}} \\
\overline{0} \\
\overline{0}\end{array}$ \\
\hline 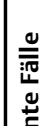 & 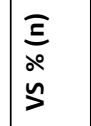 & 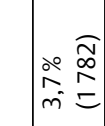 & 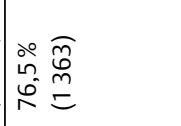 & $\mid \begin{array}{l}n \\
\hat{0} \\
6 \\
o \\
\sigma\end{array}$ & $\begin{array}{ll}\stackrel{0}{0} & \bar{\sigma} \\
0 & \bar{\sigma} \\
0 & \underline{n}\end{array}$ & 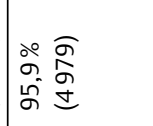 & \begin{tabular}{|l}
$\tilde{N}$ \\
$\hat{\sigma}$ \\
$\sigma$ \\
$\sigma$
\end{tabular} & 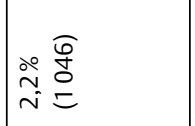 & 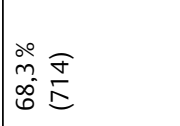 & 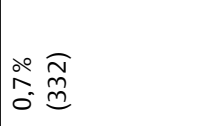 \\
\hline 疍 & 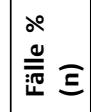 & 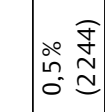 & $\begin{array}{l}\stackrel{0}{a} \\
\stackrel{0}{i n} \\
\bar{b} \stackrel{.}{=}\end{array}$ & $\begin{array}{l}0 \\
0 \\
\hat{L} \\
o \\
o \\
\sigma\end{array}$ & \begin{tabular}{ll} 
& $\widehat{o}$ \\
$\circ$ & 0 \\
0 & 0 \\
\hdashline & 0
\end{tabular} & 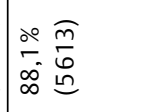 & $\begin{array}{l}\text { N } \\
\text { O } \\
\text { Oे } \\
\text { m }\end{array}$ & 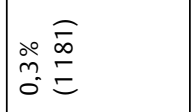 & 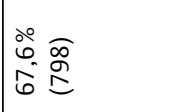 & $\begin{array}{l}\stackrel{0}{\widehat{0}} \\
\overline{0} \\
0.0\end{array}$ \\
\hline 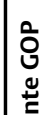 & $\stackrel{\circ}{\check{>}} \widehat{\Xi}$ & 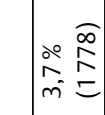 & 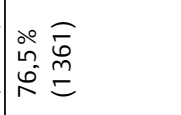 & \begin{tabular}{|l}
$\overline{\hat{n}}$ \\
$\dot{0}$ \\
$\sigma$
\end{tabular} & $\begin{array}{l}\stackrel{\frac{m}{m}}{0} \\
\dot{0} \\
\dot{0}\end{array}$ & $\begin{array}{l}\stackrel{0}{a} \\
\stackrel{\infty}{0} \\
\hat{\sigma} \\
\bar{n}\end{array}$ & 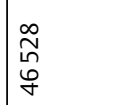 & 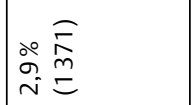 & 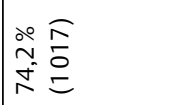 & 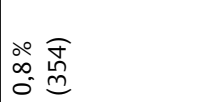 \\
\hline 言 & 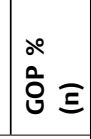 & 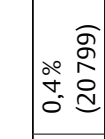 & 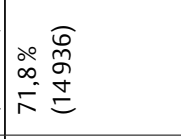 & 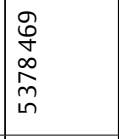 & 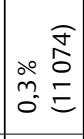 & 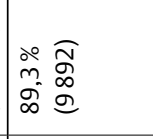 & $\begin{array}{l}\tilde{\sigma} \\
\tilde{m} \\
\tilde{m} \\
\sigma\end{array}$ & 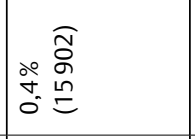 & 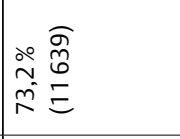 & 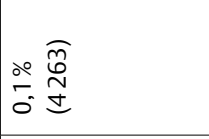 \\
\hline \multirow{2}{*}{\multicolumn{2}{|c|}{ 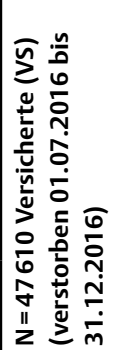 }} & 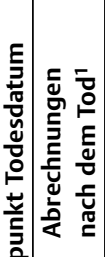 & 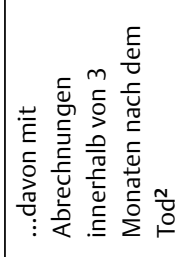 & 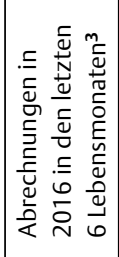 & 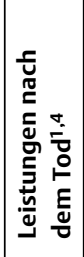 & 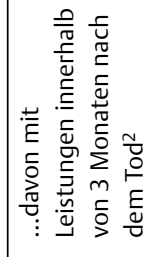 & 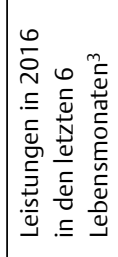 & 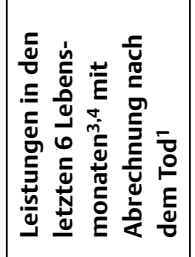 & 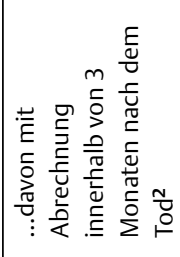 & 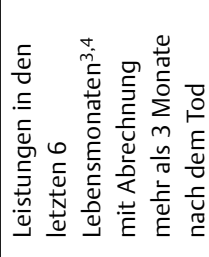 \\
\hline & & 䍖| & $\stackrel{\sim}{-}$ & $\stackrel{m}{r}$ & $\dot{N}$ & $\stackrel{\sim}{\sim}$ & $m$ & $\bar{q}$ & $\stackrel{\sim}{\sim}$ & $\stackrel{m}{\sim}$ \\
\hline
\end{tabular}




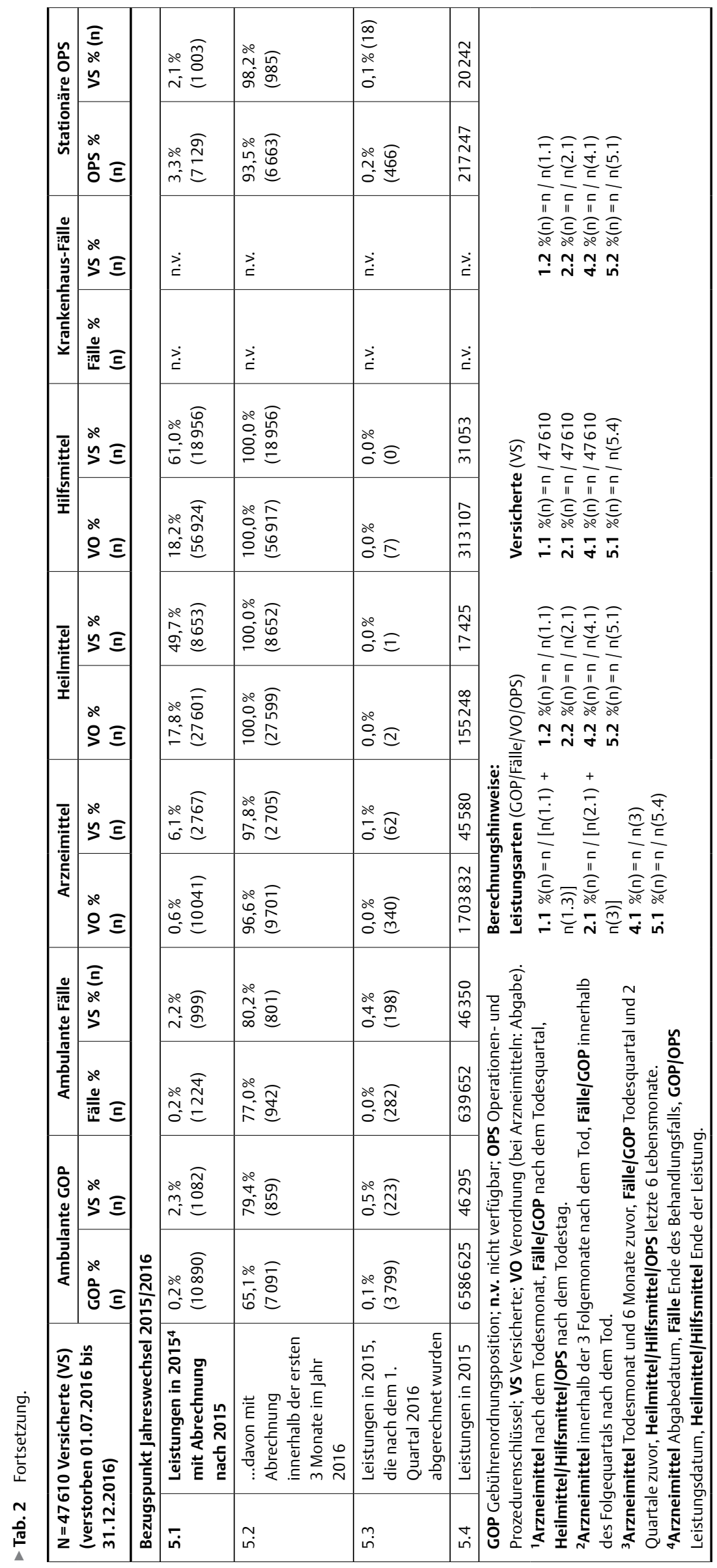


bereichen deutlich: 18,2\% der Hilfsmittel, 17,8\% der Heilmittel, 3,3\% der stationären OPS, 0,6\% der Arzneimittel-VO und jeweils $0,2 \%$ der ambulanten Fälle und der ambulanten GOP wurden in 2015 erbracht, aber erst nach dem Jahresende abgerechnet. Bezogen auf unsere Studienpopulation betraf dies zwischen 2,1\% (stationäre OPS) und 61,0\% (Hilfsmittel) der Versicherten, die eine Leistung aus dem jeweiligen Bereich im Jahr 2015 in Anspruch genommen haben ( $\triangleright$ Tab. 2, Zeile 5.1). Wieder wurde der Großteil der Leistungen innerhalb der ersten 3 Monate in 2016 abgerechnet ( Tab. 2, Zeile 5.2). Nach dem ersten Quartal 2016 wurden noch $0,2 \%$ der stationären OPS und weniger als $0,1 \%$ der Leistungen aus der ambulanten vertragsärztlichen Versorgung und der Arzneimittel-VO abgerechnet, aber nahezu keine Heil- und Hilfsmittel-VO mehr ( $\triangleright$ Tab. 2, Zeile 5.3).

\section{Diskussion}

Die Auswertung zeigt, dass im Sinne der Fragestellung relevante Abweichungen von Leistungs- und Abrechnungsdatum in den Daten aller betrachteten Leistungsbereiche vorkommen (ausgenommen Krankenhausfälle, für die diese Betrachtung anhand unserer Daten nicht möglich war).

Ein Zeitraum von 3 Monaten bzw. einem Quartal nach einem Indexereignis (hier der Tod) erscheint in den meisten Leistungsbereichen ausreichend, um die in Anspruch genommenen Leistungen nahezu vollständig in den Abrechnungsdaten vorzufinden. Lediglich bei Heil- und Hilfsmitteln sollte ggf. ein größeres Zeitintervall für die Abrechnung der Leistungen berücksichtigt werden. Der Einfluss der Abweichungen zwischen Leistungs- und Abrechnungsdatum in Bezug auf ein Indexereignis ist auch abhängig von der Studienpopulation und dem betrachteten Ereignis. So können diese weniger relevant sein für Fragestellungen wie z. B. die Inanspruchnahme einer Studienpopulation vor und nach Erstdiagnose einer bestimmten Erkrankung, aber hochrelevant für die Inanspruchnahme vor dem Tod.

In Bezug zum Jahreswechsel (hier 2015 auf 2016) sind die relativen Abweichungen insgesamt geringer. Heil- und Hilfsmittel-VO aus 2015 wurden nahezu vollständig im ersten Quartal 2016 abgerechnet. Lediglich wenige stationäre OPS aus 2015 wurden erst im zweiten Quartal 2016 abgerechnet. Diese Prozeduren erfolgen bei Krankenhausfällen, die über den Jahreswechsel verliefen und erst nach dem ersten Quartal 2016 enden. Berücksichtigt man die zeitliche Verzögerung zwischen Leistung und Abrechnung hier nicht, kann dies zu einer Unterschätzung der Inanspruchnahme führen. Insbesondere dann, wenn die datenbereitstellende Krankenkasse die Leistungen nicht nach dem Leistungsdatum, sondern nach dem Abrechnungsdatum den jeweiligen Datenjahren zuordnet. Die für den Beobachtungszeitraum bereitgestellten Daten enthalten dann die innerhalb dessen geleisteten, aber erst danach abgerechneten Leistungen nicht ( $\triangleright$ Abb. 1). Zur Überschätzung kommt es dann, wenn fälschlicherweise Leistungen aufgrund der Zuordnung über das Abrechnungsdatum dem Beobachtungszeitraum zugeordnet werden, obwohl diese früher geleistet wurden ( Abb. 1).

Nicht unerwähnt bleiben darf, dass die Auswertung folgenden Einschränkungen unterliegt: Für den Zeitpunkt der Leistungserbringung lag in allen betrachteten Leistungsbereichen ein tagesgenaues Datum vor. Für den Zeitpunkt der Abrechnung war dies nur bei den Heil- und Hilfsmitteln der Fall. Für Arzneimittel lag der Abrechnungsmonat und für ambulante Fälle und ambulante GOP das Abrechnungsquartal vor. Für stationäre OPS konnte das Entlassungsdatum des Krankenhausfalls als Surrogat verwendet werden. Die Berechnung der Differenz zwischen Abrechnung der Leistung und Todesdatum auf Monats-(Arzneimittel) bzw. Quartalsbasis (ambulante Fälle und GOP) führt somit in unseren Analysen zu einer Unterschätzung der Anteile an Abrechnungen nach dem Tod in diesen Leistungsbereichen. Bei den Analysen zu den Leistungen nach dem Tod kann die Verwendung des Leistungsendes für Heil- und insbesondere für Hilfsmittel als Zeitpunkt der Leistungsinanspruchnahme zur Überschätzung der Inanspruchnahme nach dem Tod führen. Des Weiteren ist zu berücksichtigen, dass das Todesdatum als abgeleitetes Datenfeld aus dem Datum des Versicherungsendes mit dem Tod als Austrittsgrund aus der Krankenkasse einer gewissen Unsicherheit unterliegt. Generell ist davon auszugehen, dass der Austrittsgrund Tod sehr valide dokumentiert ist; eine höhere Wahrscheinlichkeit von Fehleinträgen besteht bei Mitversicherten [4]. Bei Horenkamp-Sonntag et al. wird das Vorhandensein von Abrechnungsfällen/Leistungen nach dem Tod generell als möglicher Hinweis auf Datenfehler beim Todesdatum genannt [3]. Eine sehr weitgehende Konsequenz dieser Überlegung wäre dann der Ausschluss aller Versicherten aus der Analyse zur Inanspruchnahme vor dem Tod. Im Folgenden legen wir dar, dass es nachvollziehbare Gründe für Abrechnungsfälle und Leistungen nach dem Tod gibt, weshalb deren Vorhandensein nicht ausreichend begründet, das Todesdatum anzuzweifeln.

Für alle Leistungsbereiche mit mehrstufigen Erfassungsvorgängen in der Datenverarbeitung, wie bspw. die Übertragung ambulanter vertragsärztlicher Leistungen durch die Kassenärztlichen Vereinigungen (KVen) an die Krankenkassen, ist eine gewisse Fehleranfälligkeit anzunehmen [3]. Bei ambulanten Arzneimittel-VO können z. B. Datenverarbeitungsfehler beim Einscannen und der digitalen Schrifterkennung der Arzneimittelrezepte auftreten [3] Die Fehlerwahrscheinlichkeit steigt auch mit der Änderung von Abrechnungsregeln [3].

Weiter existieren für ambulante Abrechnungen vielfach feste Abrechnungsintervalle. So erfolgen z. B. vertragsärztliche Abrechnungen zum Quartalsende, Abrechnungen für Heilmittel hingegen monatlich [5, 6]. Dies kann Auswirkungen auf Zeitdifferenzen sowohl zwischen Tod und Inanspruchnahme als auch zwischen Tod und Abrechnung haben: Für die Inanspruchnahme ist zu vermuten, dass Leistungserbringer mit Erstellen der Abrechnung bis dahin nicht dokumentierte Leistungen mit dem Abrechnungsdatum anstelle des ursprünglichen Leistungsdatums dokumentieren. Leistungen mit einem Datum der Inanspruchnahme nach dem Todesdatum können so zu einem Großteil im Monat bzw. Quartal des Versterbens erfolgen. Dies bestätigt sich in einer zusätzlichen Analyse: bei 80,8\% der ambulanten GOP mit einem Leistungsdatum nach dem Todesdatum erfolgte die Leistung innerhalb des Todesquartals des Versicherten (Daten nicht dargestellt). Für ambulante Behandlungsfälle und GOP besteht außerdem die Möglichkeit, aufgrund individueller Abrechnungsbestimmungen der KVen, Behandlungsfälle aus vorangegangenen Quartalen in begründeten Einzelfällen bis zum Ablauf einer KV-spezifischen Frist nach Inanspruchnahme als sogenannte „Nachzüglerfälle“ abzurechnen [5, 7]. 
Ein weiterer Erklärungsansatz für eine Inanspruchnahme nach dem Tod ist die Auslösung einer Leistung durch eine bevollmächtigte Person des Versicherten (z. B. Angehöriger oder ambulanter Pflegedienst), ggf. in Unkenntnis des zwischenzeitlich eingetretenen Todes des Versicherten. Beispiele hierfür sind das Einlösen eines Arzneimittelrezeptes oder die Quittierung des Empfangs von Hilfsmitteln. Bei Hilfsmittel-VO zeigte sich in unseren Analysen ein hoher Anteil an Leistungen nach dem Tod. Eine denkbare Erklärung ist die zeitliche Verzögerung bei der Rückgabe von Hilfsmitteln nach dem Versterben eines Versicherten.

Für die Leistungsinanspruchnahme nach dem Tod bei Krankenhausfällen und stationären OPS ist am ehesten von einer tatsächlich fehlerhaften Dokumentation auszugehen. Insgesamt kann davon ausgegangen werden, dass die intensivere Kontrolle von Krankenhausabrechnungen zu der deutlich geringeren Zahl an Abweichungen beiträgt.

Zeigen sich über mehrere Sektoren hinweg Leistungen mit einem Datum nach dem Todesdatum, kann auch ein Fehler im Todesdatum die Ursache sein. So kann es z. B. bei der Übermittlung des Todesdatums an die GKV zu Fehlern kommen. Bei vereinzelt auftretenden Fällen extremer Abweichung, bspw. mit einem Leistungsdatum mehr als 2 Jahre nach dem Todesdatum, ist auch eine wiederholte Vergabe eines Versichertenpseudonyms innerhalb der Studienpopulation als mögliche Erklärung in Betracht zu ziehen.

Diese Erklärungsansätze zeigen, dass Abrechnungen bzw. Leistungen nach dem Tod einerseits auf erlaubte und nachvollziehbare Dokumentations- und Abrechnungsmodalitäten zurückgeführt werden können, andererseits aber auch Folgen von Dokumentationsbzw. Datenverarbeitungsfehlern sein können. Korrekturdatenlieferungen sind bei inhaltlichen Fehlern in den Abrechnungsdaten im Datenaustausch zwischen den KVen und den Krankenkassen nicht zwingend vorgesehen [3]. Bei der Arbeit mit vorstrukturierten Daten eines WDWH ist außerdem zu beachten, dass hier ggf. nur einmalige Datenabzüge aus dem produktiven Datawarehouse erfolgen. Später stattfindende Datenkorrekturen wirken sich nicht auf die Inhalte des WDWH aus.

Zusammenfassend ist festzustellen, dass die Erklärungen für Leistungen und Abrechnungen nach dem Tod nur ansatzweise erfolgen konnten. Hier sehen wir weiteren Forschungsbedarf: Dieser betrifft einerseits die Deskription der Auftrittshäufigkeit in unterschiedlichen Datenquellen und auch in unterschiedlichen Jahren. Andererseits könnte mittels qualitativer Studien ein besseres Verständnis der Entstehungs- und Verarbeitungsprozesse von GKV-Routinedaten erreicht werden. Eine geeignete Methode wäre die Durchführung von Interviews mit Vertretern aus den einzelnen Schritten der Datenverarbeitung von der Kodierung in der Versorgungseinrichtung, über die Abrechnungsverarbeitung bei den KVen und Krankenkassen, bei den in die Datenverarbeitung einbezogenen Dienstleistern bis hin zu den Rechenzentren bzw. Datawarehouses, über die die Daten für Forschungszwecke zur Verfügung gestellt werden. Auf diesem Weg können die zu den gezeigten Abweichungen führenden Umstände nachvollziehbar gemacht und angemessenere Lösungsansätze entwickelt werden. Auch lassen sich aus so gewonnenen Informationen dann möglicherweise exaktere Validierungsstrategien für das Todesdatum in den GKV-Daten ableiten.

\section{FAZIT FÜR DIE PRAXIS}

Für alle Routinedatenauswertungen zur Leistungsinanspruchnahme können die beobachteten Abweichungen von Leistungs- und Abrechnungsdatum nicht ignoriert werden, da diese zur Über- oder Unterschätzung der Leistungsinanspruchnahme oder auch zu Fehlinterpretationen hinsichtlich Beginn, Dauer und Ende von Versorgung führen können.

Entscheidend ist, dass die gewählte Strategie im Umgang mit diesen Abweichungen im Einklang mit den Forschungszielen steht. Dabei sind aus unserer Sicht 2 Fragen hilfreich: „Wie schwerwiegend sind die auftretenden Probleme mit Blick auf die Forschungsfragen?“ und „Was sind die Auswirkungen der verschiedenen Lösungsansätze?“ Werden z. B. Gruppen von Versicherten verglichen, die von den Abweichungen gleichermaßen betroffen sind, dürfte die Auswirkung auf das Ergebnis eher geringfügig sein. Dies gilt ggf. auch, wenn jährliche Inanspruchnahme-Kennzahlen ermittelt werden sollen und die Abweichungen am Anfang und Ende des Beobachtungsjahres gleichermaßen wirken. Geht es jedoch um die Analyse der Inanspruchnahme bis zum Tod (bzw. einem alternativen datumsbezogenen Ereignis), kann das Ergebnis derart verfälscht sein, dass inhaltliche Fehlinterpretationen resultieren können. Auch bei Projekten, die eine Zeitreihenerfassung vorsehen, sollten diese Abweichungen für die Festlegung der (frühesten und spätesten) Datenlieferzeitpunkte berücksichtigt werden.

Ein pragmatischer Umgang mit Leistungen, deren Leistungs- bzw. Abrechnungsdatum nach dem Tod liegen, besteht darin, diese als erlaubt zu betrachten und somit konsequent zu berücksichtigen. Bei projektbezogenen Datenlieferungen kann eine entsprechende Spezifikation in der Datenanforderung erfolgen, wobei der zeitliche Verzug zwischen Leistungs- und Abrechnungsdatum explizit berücksichtigt werden muss. Dabei kann bei länger zurückliegenden Daten die Vollständigkeit eher erreicht werden als bei sehr aktuellen Daten. Wurde eine entsprechend ausgedehnte Datenlieferung versäumt, ist eine erneute, korrigierte Datenanforderung anzustreben. Bei bereits vorstrukturierten Daten (z. B. WDWH) kann eine Erweiterung der einzuschließenden Zeiträume, um mindestens 1, besser 2 Quartale nach Ende des Betrachtungszeitraums helfen, die aufgezeigten zeitlichen Differenzen bei der Abrechnung von Leistungen zu berücksichtigen. In jedem Fall sollten die beschriebenen Abweichungen aber im Zuge der Plausibilitätsprüfung nach der Datenlieferung abgeklärt werden. Das entspricht auch den gängigen Empfehlungen zur Sekundärdatenanalyse [2,8]. Eine differenziertere aber aufwändigere Art, mit den Datumsabweichungen umzugehen, besteht darin, nicht plausible Datensätze bzw. Versicherte auszuschließen. In jedem Fall ist bei der Veröffentlichung bzw. Darstellung der Ergebnisse darauf zu achten, dass im Sinne der Standardisierten Berichtsroutine für Sekundärdaten Analysen (STROSA) der Umgang mit den Daten (beispielsweise das Zulassen von Leistungen nach dem Tod) zu berichten ist [9]. 


\section{Interessenkonflikt}

Die Autorinnen/Autoren geben an, dass kein Interessenkonflikt besteht.

\section{Literatur}

[1] AQUA Allgemeine Spezifikation für die Nutzung der Sozialdaten bei den Krankenkassen - Projektabschlussbericht (Stand: 31. März 2014). AQUA - Institut für angewandte Qualitätsförderung und Forschung im Gesundheitswesen GmbH. Göttingen. 2014

[2] Swart E, Gothe H, Geyer S et al. Gute Praxis Sekundärdatenanalyse (GPS): Leitlinien und Empfehlungen. 3. Fassung; Version 2012/2014. Gesundheitswesen 2015; 77: 120-126. doi:10.1055/s-0034-1396815

[3] Horenkamp-Sonntag D, Lindern R, Wenzel F et al. Prüfung der Datenqualität und Validität von GKV-Routinedaten. In: Swart E, Ihle P, Gothe H, Matusiewicz D. Routinedaten im Gesundheitswesen. 2. Auflage. Bern: Verlag Hans Huber. 2014: 314-330

[4] Scholz R, Sauer S, Müller R. Analysen zur Sterblichkeit. In: Swart E, Ihle P, Gothe H, Matusiewicz D. Routinedaten im Gesundheitswesen. 2. Auflage Bern: Verlag Hans Huber; 2014: 38-42
[5] Kassenärztlichen Vereinigung Thüringen Abrechnungsrichtlinien der Kassenärztlichen Vereinigung Thüringen gültig ab 01.04.2012, gemäß $\S 11$ Absatz 4 (i) der Satzung der KV Thüringen. Im Internet: https:// www.kv-thueringen.de/mitglieder/abr_hon/10_la/60_abrrlkvt/10_ AbrechnungsRL_2Q2012.pdf; Stand: 29.11.2018

[6] Verband der Ersatzkasse e.V (vdek). Rahmenvertrag der Berufsverbände Physiotherapie und dem Verband der Ersatzkassen e.V. (vdek) vom 01.04.2013. Im Internet: https://www.vdek.com/vertragspartner/ heilmittel/rahmenvertrag/_jcr_content/par/download_14/file.res/ RV_2013_UF.pdf; Stand: 29.11.2018

[7] Kassenärztliche Vereinigung Saarland Abrechnungsbestimmungen der KV Saarland, ab 01.07.2016, Version 4 It. VV-Beschluss vom 15.06.2018. Im Internet: https://www.kvsaarland.de/documents/ 10184/480827/Abrechnungsbestimmungen+2016; Stand: 29.11.2018

[8] Swart E, Ihle P, Gothe $\mathrm{H}$ et al. Routinedaten im Gesundheitswesen. 2. Auflage Bern: Verlag Hans Huber; 2014

[9] Swart E, Blitzer EM, Gothe $\mathrm{H}$ et al. STandardisierte BerichtsROutine für Sekundärdaten Analysen (STROSA) - ein konsentierter Berichtsstandard für Deutschland, Version 2. Gesundheitswesen 2016; 78: (Suppl. 01) e145-e160. doi:10.1055/s-0042-108647 